MISS ANDREA CAMPOS-CANDELA (Orcid ID : 0000-0003-0827-3890)

Article type : Research Article

Handling Editor: Jean-Michel Gaillard

\title{
A camera-based method for estimating absolute density in animals displaying home range behaviour
}

\author{
Andrea Campos-Candela ${ }^{* \mathbf{a} b}$, Miquel Palmer ${ }^{\mathbf{a}}$, Salvador Balle ${ }^{\mathbf{c}}$ and Josep \\ Alós ${ }^{a}$ \\ a \\ Department of Ecology and Marine Resources, Institut Mediterrani d’Estudis Avançats, \\ IMEDEA (CSIC-UIB), C/ Miquel Marquès 21, 07190 Esporles, Balearic Islands, Spain

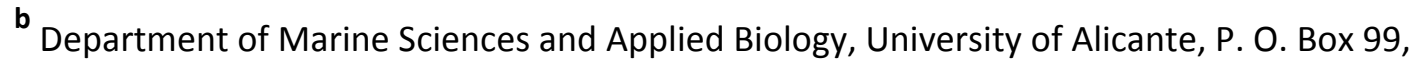 \\ 03080 Alicante, Spain \\ c Department of Marine Technologies, Operational Oceanography and Sustainability, Institut \\ Mediterrani d'Estudis Avançats, IMEDEA (CSIC-UIB), C/ Miquel Marquès 21, 07190 Esporles, \\ Balearic Islands, Spain \\ *Corresponding author: acampos@imedea.uib-csic.es
}

Running headline: Estimating density of animals with home range.

This article has been accepted for publication and undergone full peer review but has not been through the copyediting, typesetting, pagination and proofreading process, which may lead to differences between this version and the Version of Record. Please cite this article as doi: 10.1111/1365-2656.12787

This article is protected by copyright. All rights reserved. 


\section{Summary}

1. The measurement of animal density may take advantage of recent technological achievements in wildlife video recording. Fostering the theoretical links between the patterns depicted by cameras and absolute density is required to exploit this potential.

2. We explore the applicability of the Hutchinson-Waser's postulate (i.e., when animal density is stationary at a given temporal and spatial scale, the absolute density is given by the average number of animals counted per frame), which is a counter-intuitive statement for most ecologists and managers who are concerned with counting the same individual more than once. We aimed to reconcile such skepticism for animals displaying home range behaviour.

3. The specific objectives of this paper are to generalize the Hutchinson-Waser's postulate for animals displaying home range behaviour and to propose a Bayesian implementation to estimate density from counts per frame using video cameras.

4. Accuracy and precision of the method was evaluated by means of computer simulation experiments. Specifically, six animal archetypes displaying well-contrasted movement features were considered. The simulation results demonstrate that density could be accurately estimated after an affordable sampling effort (i.e., number of cameras and deployment time) for a great number of animals across taxa.

5. The proposed method may complement other conventional methods for estimating animal density. The major advantages are that identifying an animal at the individual level and precise knowledge on how animals move are not needed, and that density can be estimated in a single survey. The method can accommodate conventional camera trapping data. The major limitations are related to some implicit assumptions of the underlying model: the home range centres should be homogeneously distributed, the detection probability within the area surveyed by the camera should be known, and animals should move independently to one another. Further improvements for circumventing these limitations are discussed.

\section{Key words}

Abundance, biased random walk (BRW), counts, movement, space occupancy, speed, terrestrial and aquatic wildlife surveys, video recording

This article is protected by copyright. All rights reserved. 


\section{Introduction}

How to accurately and precisely measure animal density (i.e., the number of animals per unit area) is a long-standing but not fully resolved question (Dénes, Silveira \& Beissinger 2015). With the recent technological achievements in wildlife video recording, managers and ecologists have gained powerful tools for improving terrestrial and aquatic wildlife surveys, which typically target animal distribution, abundance or behaviour (Burton et al. 2015). For example, unmanned aerial vehicles (UAVs) are currently used for a wide range of wildlife monitoring applications (Linchant et al. 2015), covering birds (Vas et al. 2015) to terrestrial and aquatic mammals (Martin et al. 2012; Hodgson et al. 2016). In aquatic systems, the recent miniaturization and cost reduction of underwater video recording devices and the installation of cabled video observatories have broadened the remote, long-term and high-frequency monitoring of fish and their environments (Aguzzi et al., 2015; Matabos et al. 2015). An additional advantage of camera devices is the minimization of wildlife disturbance (Assis et al. 2013; Vas et al. 2015) and researcher risk linked to surveying under difficult conditions and in hard-to-reach populations or areas (Martin et al. 2012; Hodgson et al. 2016; Goebel et al. 2015). Overall, changing from conventional survey methods (e.g., physical mark-and-recapture surveys, visual censuses, trammel-net surveys, etc.) to camera-based wildlife assessment may represent a paradigm change in the current technological era. For example, photoidentification (Moya et al. 2015) may become the input for spatial capture-recapture models (Chandler \& Royle 2013). Concerning marine wildlife assessment, conventional methods (e.g., underwater visual census) are currently being complemented with data from underwater cameras (see Mallet and Pelletier (2014) for a review).

The technological advances in wildlife imaging have opened at least two new challenges and opportunities: developing novel algorithms for data mining and automated image interpretation (Aguzzi et al. 2015, Boom et al. 2014, Díaz-Gil et al. 2017; reviewed in Dell et al. 2014) and fostering theoretical links between the patterns depicted by cameras and absolute animal density. Here, we focus on this second challenge because there continues to be debate on which method and camera metric should be preferred. Most of the proposed methods can be classified into two broad categories: empirical approaches that aim to correlate a given camera metric with independent estimates of density (e.g., Pelletier et al. 2011; Bacheler et al. 2013; Schobernd et al. 2014; Campbell et al. 2015) and theoretical approaches that exploit different model-derived expectations regarding how animals move

This article is protected by copyright. All rights reserved. 
and thus how they are expected to intercept a camera (e.g., Rowcliffe et al. 2008; Keeping \& Pelletier 2014; Boschetti \& Vanderklift 2015; Lucas et al. 2015).

Most of the theoretical contributions are based on the encounter concept, which is defined as any non-instantaneous event during which an animal is continuously detected by a camera. The encounter rate (i.e., the number of encounters per unit time) depends not only on the animal density but also on the way the animal moves (Hutchinson \& Waser 2007; Rowcliffe et al. 2008; Glennie, Buckland \& Thomas 2015). This dependency imposes severe difficulties in practice. For example, the definition and measurement of speed is an elusive topic (e.g., average measures of foraging, maximum, critical or cruising speed by telemetry, forced swim tests, camera traps or GPS telemetry, among others), and accurate and standardized data on animal speed are relatively scarce. This scarcity is particularly apparent among aquatic animals (Boschetti \& Vanderklift 2015), although see Hussey et al. (2015) for improvements.

Fortunately, the speed dependency of encounters may be circumvented using an alternative metric: the association (Hutchinson \& Waser 2007), which is defined as the number of ongoing occurrences within a given area and at a given instant. In terms of a camera survey, this metric may be translated as the number of animals counted within a given area in a given frame. According to Hutchinson \& Waser (2007), the absolute animal density can be properly estimated from the averaged counts across $n$ independent frames divided by the area actually surveyed by the camera, under the assumption that animal density is stationary at a given temporal and spatial scale. This unexpectedly simple postulate may seem quite counterintuitive for most ecologists and managers when considering the pros and cons of implementing a camera-based wildlife monitoring program because most methods are typically concerned with counting the same individual more than once (Ward-Paige, Mills Flemming \& Lotze 2010; Campbell et al. 2015). This assessment is reinforced by the fact that many animals remain within a given area or home range (hereafter HR) most of the time, which is usually smaller than the extent of suitable habitat (Börger, Dalziel \& Fryxell 2008; Kie et al. 2010; Owen-Smith \& Martin 2015; Tao, Börger \& Hastings 2016). Certainly, in these cases, the probability of counting the same animal in several frames is non-negligible. This property is actually exploited in spatial capture-recapture models that explicitly require the detection of the same individual more than once and in more than one site (Chandler \& Royle 2013).

In order to reconcile such skepticism, this work aims to demonstrate that animal density can be effectively estimated from animal counts averaged across several frames of a video survey if the probability density function of finding an animal in a given location is stationary, which is exactly the case for animals displaying home range (HR) behaviour. To

This article is protected by copyright. All rights reserved. 
achieve this objective, we first introduce a movement model that describes a HR pattern of space occupancy with radial symmetry (as an approximation). Second, we generalize the Hutchinson-Waser's postulate for any movement model leading to a HR pattern of space occupancy. Finally, several computer simulation experiments consisting of moving animals according to realistic (empirically estimated) movement parameters in an arena of known density are implemented, which allowed to assess the reliability of the method for obtaining accurate and precise density estimates after a sensible sampling effort (i.e., number of cameras and deployment time). Sensibility analysis are completed using six well-contrasted animal archetypes that were selected across taxa according to a number of variables that beforehand were presumed to affect the accuracy and precision of absolute density estimates from video surveys (body mass, size of the HR, speed and density). The limits of the method and the suitability of the implicit assumptions are discussed.

\section{Materials and Methods}

\section{HR space occupancy pattern as described by a Langevin process}

Several mechanistic models are able to reproduce the spatial patterns displayed by free-ranging animals, which consist of remaining within a relatively small area or HR most of the time (Börger et al. 2008; Van Moorter et al. 2009). Among them, for simplicity and convenience, we considered the biased random walk (BRW), which can be mathematically described as a special case of the Langevin equation (Gardiner 1990). Nevertheless, the derivation of the expected number of animals counted in a given frame (see below) is general for any movement model that results in a HR pattern.

According to Dubkov and Spagnolo (2007), the Langevin equation is given by:

$$
\frac{\mathrm{d} \vec{x}}{\mathrm{~d} t}=-\vec{\nabla} U(\vec{x})+\sqrt{\varepsilon} \vec{\xi}(t) \quad \text { eqn } 1
$$

where $\vec{x}(t)$ denotes the particle displacement. This formulation splits a deterministic force term (i.e., the potential field within which a particle is moving), $\vec{\nabla} \mathrm{U}(\vec{x})$, from a stochastic stimulus term, $\sqrt{\varepsilon} \vec{\xi}(t)$, which is Gaussian-distributed (white noise) with zero mean, zero covariance between the two dimensions (i.e., spatial coordinates), and the same variance $(\varepsilon)$ in each spatial dimension. Concerning the deterministic force, the simplest choice for the case of a HR pattern is a harmonic, time-invariant, potential field given by:

$$
\vec{\nabla} \mathrm{U}(\vec{x})=k \vec{x}
$$

where $k$ denotes the strength of the harmonic force.

This article is protected by copyright. All rights reserved. 
The biological rationale behind this model is that animals move within a homogeneous environment following random stimuli with an additional rule that determines a tendency (drift) to remain around a specific point (the HR centre). The emerging pattern is a circular HR (Palmer et al. 2011).

The numerical approximation of eqn 1 to the real trajectory sampled with a finite time step, $\Delta t$, can be described according to Alós et al. (2016) by:

$$
\vec{r}_{\mathrm{n}+1}=\vec{r}_{\mathrm{c}}+\mathrm{e}^{-\mathrm{k} \Delta \mathrm{t}}\left(\vec{r}_{\mathrm{n}}-\vec{r}_{\mathrm{c}}\right)+\vec{R}_{\mathrm{n}}
$$

eqn 3

where $\vec{r}_{\mathrm{n}}$ denotes the position of the animal at time $t_{\mathrm{n}}=n \Delta t$ ( $n$ being an integer), $\vec{r}_{\mathrm{c}}$ is the position of the center of the HR, $k$ is the central harmonic constant force attracting the animal toward $\vec{r}_{\mathrm{c}}$ (eqn 2), and $\vec{R}_{\mathrm{n}}$ is a stochastic term, normally distributed with zero mean and standard deviation in each dimension approximated according to Palmer et al. (2011) by:

$$
\sigma=\sqrt{\frac{\varepsilon\left(1-\mathrm{e}^{-2 \mathrm{k} \Delta \mathrm{t}}\right)}{2 k}}
$$

eqn 4

where $\varepsilon$ is the variance of $\vec{\xi}$ in eqn 1 .

Provided that $k$ and $\varepsilon$ are difficult to interpret on the biological side, two biologically interpretable parameters (radius and speed) have been derived from them. The radius of the circular HR (area within which an animal has a 95\% probability of being found when a large period of time is considered) can be approximated according to Palmer et al. (2011) by:

$$
\text { radius }=\sqrt{-\frac{\varepsilon \ln (1-0.95)}{k}} \quad \text { eqn } 5
$$

Similarly, speed can be defined as the square root of the averaged squared distance travelled from $n$ to $n+1$ :

$$
\text { speed }=\frac{1}{\Delta t} \sqrt{\left\langle\left|\vec{r}_{\mathrm{n}+1}-\vec{r}_{\mathrm{n}}\right|^{2}\right\rangle}=\frac{1}{\Delta t} \sqrt{\varepsilon \frac{1-\mathrm{e}^{-2 \mathrm{k} \Delta \mathrm{t}}}{k}} \quad \text { eqn } 6
$$

Note, however, that eqn 3 will be a reasonable approximation whenever the product $\mathrm{k}^{*} \Delta t$ is small enough (e.g., 0.1). In that case,

$$
\text { speed }=\frac{k}{0.1} \sqrt{\varepsilon \frac{1-\mathrm{e}^{-0.2}}{k}}=\sqrt{\varepsilon k \frac{1-\mathrm{e}^{-0.2}}{0.1^{2}}} \propto \sqrt{\varepsilon k} \quad \text { eqn } 7
$$

\section{Derivation of Hutchinson-Waser's postulate for any animal displaying HR behaviour}

We derive the Hutchinson-Waser's postulate according to the following assumptions:

(i) A given camera detects (detection probability $=1$ ) any animal within an area measured with negligible error (detection area).

(ii) Animals move independently from one another.

This article is protected by copyright. All rights reserved. 
(iii) Animals display a HR space occupancy pattern, and the HR centres are randomly and homogeneously distributed.

The third assumption is particularly relevant. Note that strictly speaking, animal density is time- varying when it is naively (i.e., wrongly) defined as the number of individuals counted in a given area and at a given moment because animals move, and some of them may be continuously entering or exiting the area considered. However, when animals display HR behaviour, and according to eqn 1 , a stationary probability density function, $P_{\mathrm{st}}\left(\vec{r} \mid \vec{r}_{\mathrm{c}}\right)$, of finding the animal at the position $\vec{r}$ exists, provided that the HR centre is located at $\vec{r}_{\mathrm{c}}$ (Dubkov \& Spagnolo 2007). This fact implies that under assumption (iii), animal density (thereafter, the density of the HR centres) is stationary at the spatial and temporal scales of the measurement period. In fact, the existence of $P_{\mathrm{st}}$ is independent of the nature of the stochastic term, $\vec{\xi}(t)$, in eqn 1 and guarantees that the underlying dynamic system is ergodic. Ergodicity ensures the equivalence of constructing $P_{\mathrm{st}}$ either by recording over infinite time the positions of a single animal or by averaging over space the positions of infinite (identical, in terms of HR size, HR centre and exploration rate) animals.

When assumptions (i) and (ii) are met and $P_{\mathrm{st}}$ exists, the probability $\pi\left(\vec{r}_{\mathrm{c}}\right)$ that the camera (located at the origin for the sake of simplicity) detects an animal having its HR centre at $\vec{r}_{\mathrm{c}}$ is given by the probability density that the individual is located at $\vec{r}$, conditional to the probability that the HR centre of this individual is at $\vec{r}_{\mathrm{c}},\left(P_{\mathrm{st}}\left(\vec{r} \mid \vec{r}_{\mathrm{c}}\right)\right)$, times the probability density of detection of the individual located at $\vec{r}, P_{\mathrm{d}}(\vec{r})$, integrated over the detection area, $\mathrm{Z}$, of the camera:

$$
\pi\left(\vec{r}_{\mathrm{c}}\right)=\int_{\mathrm{Z}} P_{\mathrm{d}}(\vec{r}) P_{\mathrm{st}}\left(\vec{r} \mid \vec{r}_{\mathrm{c}}\right) \mathrm{d} \vec{r}
$$

Thus, the number of animals per frame that the camera detects is given by a Poisson distribution with mean:

$$
<N_{\text {obs }}>=\int_{\infty} D\left(\vec{r}_{\mathrm{c}}\right) \pi\left(\vec{r}_{\mathrm{c}}\right) \mathrm{d} \vec{r}_{\mathrm{c}}
$$

where $D\left(\vec{r}_{\mathrm{c}}\right)$ denotes the density of the HR centres and the surface integral over $\vec{r}_{\mathrm{c}}$ extends to the whole space. In the specific case of a homogeneous distribution (assumption iii), and since $P_{\mathrm{st}}\left(\vec{r} \mid \vec{r}_{\mathrm{c}}\right)=P_{s t}\left(\vec{r}-\vec{r}_{\mathrm{c}}\right)$, the integral over $\vec{r}_{\mathrm{c}}$ yields 1 , hence,

$$
<N_{\text {obs }}>=D \int_{\infty} \int_{\mathrm{Z}} P_{\mathrm{d}}(\vec{r}) P_{\mathrm{st}}\left(\vec{r} \mid \vec{r}_{\mathrm{c}}\right) \mathrm{d} \vec{r} \mathrm{~d} \vec{r}_{\mathrm{c}}=D \int_{\mathrm{Z}} P_{\mathrm{d}}(\vec{r}) \mathrm{d} \vec{r}=D Z \text { eqn } 10
$$

where $\mathrm{Z}$ is the detection area of the camera where $P_{\mathrm{d}}(\vec{r})$ is one. Thus, the observed number of counts in a given frame of the camera will be Poisson-distributed with characteristic parameter $=<N_{\text {obs }}>$.

This derivation can be extended to the case where multiple cameras are deployed to allow between-camera variability to accommodate some environmental variations or

This article is protected by copyright. All rights reserved. 
patchiness (i.e., the sites where cameras are deployed may display environmental differences, which may translate into differences in animal densities). In that case, the observed number of counts in frame $i$ of camera $j\left(N_{\mathrm{ij}}\right)$ is assumed to be Poisson-distributed around a mean of $\lambda_{\mathrm{j}}$. At an upper level, the log-transformed values of $\lambda_{j}$ are assumed to be normally distributed around a general mean of $\mu$ with a standard deviation of $s d$ (i.e., cameras are considered as a random factor):

$$
\begin{aligned}
& N_{\mathrm{ij}} \sim \operatorname{Poisson}\left(\lambda_{\mathrm{j}}\right) \\
& \log \left(\lambda_{\mathrm{j}}\right)=\mu+\delta_{\mathrm{j}} \\
& \delta_{\mathrm{j}} \sim \operatorname{Normal}(0, s d) \\
& D=e^{\mu} / \pi L^{2}
\end{aligned}
$$

where $D$ is the density of the HR centres, $L$ is the radius of the detection area of the camera, and $s d$ is the standard deviation of the between-camera differences $\left(\delta_{\mathrm{j}}\right)$. Therefore, the averaged density across cameras and its variance can be derived from the model parameters.

\section{Reliability and applicability of the method across taxa}

Computer simulation experiments have been conducted to explore the reliability and sensitivity of the proposed derivation (in terms of accuracy and precision), and its usefulness and limitations (in terms of applicability to realistic scenarios). Conventional sensibility analysis implies to explore the effects of any combination of the involved variables. Here, provided the large number of variables potentially involved in affecting the quality of density estimates and the fact that most of them are correlated (see below), instead of exploring the full universe of combinations of the range of possible values for each of the considered variables, only a few number of them representing well-differentiated animal archetypes were selected. Archetypes were defined aiming to identify the key biological features of a given animal that may affect the quality of the estimates. Therefore, the pattern depicted in the results section should be interpreted as general guide since the archetypes are only defined by the selected variables and other species-specific characteristics are ignored (see more discussion below).

\section{Selecting different animal archetypes}

A comprehensive search was conducted using Google Scholar and Web of Science to compile species of any taxa for which data on four variables that beforehand were presumed to affect the quality of density estimates (density, body mass, speed and HR size) were available. Different combinations of keywords (HR, biomass, density, abundance, movement, speed and velocity) were used. The reference list of any reference resulting from this primary search was in turn checked to identify additional data. Finally, when information for one of the

This article is protected by copyright. All rights reserved. 
four variables was lacking for a given species, an additional search was completed with the species name as a keyword. Whenever possible, details of the method used to measure speed and density were recorded since they include different sources of bias, although we use the reported data irrespective of the method..

To select well-differentiated archetypes, a principal component analysis (PCA) was conducted to density, body mass, speed and HR size using the rda function as implemented in the vegan package (Oksanen et al. 2016) of the R software. All variables were log-transformed and scaled to have unit variance prior to the PCA.

\section{Simulation settings}

Invariant settings were preferred to facilitate the archetype comparisons. Accordingly, the radius of the circular area within which any animal is detected (camera radius) was defined in a way that, on average, $m$ animals per frame are counted:

$$
\text { camera radius }=\sqrt{\frac{m}{\pi D}}
$$

where $D$ is the actual animal density of a given archetype.

A virtual camera was set at the centre of a squared scenario with side defined as:

$$
\text { side }=2 \text { camera radius }+2 \text { Radius }
$$

where Radius is the radius of the HR. The rationale of defining such a buffer ( $2 *$ Radius) is that an animal with its HR centre outside the scenario considered (and thus not included in the simulation settings) has a negligible probability of being detected by the camera. The number of animals to be moved within such a scenario is given by $\operatorname{side}^{2 *} D$.

The HR centres of the simulated animals were randomly distributed within the virtual scenario (arena), and each animal was moved according to eqn 3 . The movement parameters $k$ and $\varepsilon$ were estimated (eqns 5 and 6 ) using the data compiled as described above. Product $\Delta t^{*} k$ was set to 0.1 to ensure negligible error related to the discretization of the movement equation (eqn 3). Note that this causes the time step, $\Delta t$, to be scale invariant. Ten cameras were set in each simulation experiment. The positions of all simulated animals were actualized at each of 300 time steps. The number of animals within the area detected by the camera was recorded every 5 time steps from the $5^{\text {th }}$ through the $300^{\text {th }}$ time step (i.e., up to 60 frames per simulation experiment). Temporal autocorrelation in the counts of the same camera was tested with the acf function in the R program (http://www.r-project.org/). Up to 15 independent simulation experiments (i.e., a new set of animals was created and moved in a new scenario) were replicated for each archetype. For each of these 90 experiments, the obtained dataset was a matrix of 60 rows (i.e., count of animals detected per frame in 60 frames) and 10 columns (cameras).

This article is protected by copyright. All rights reserved. 


\section{Estimating animal density}

Animal density was estimated by a Bayesian implementation of eqn 11 using the jags function from the R2jags package (Su \& Yajima 2015). A uniform prior between -30 and 30 was set for $\mu$, which translates into densities between $9.3 \times 10^{-14}$ and $1.1 \times 10^{13}$. A gamma-distributed prior with shape $=0.001$ and rate $=0.001$ were set for tolerance, which is the squared inverse of $s d$ (eqn 11). For each dataset, five Markov chain Monte Carlo (MCMC) simulations were run. We drew 12,000 posterior samples, discarded the initial 2,000 iterations (burning period), and one out of 50 of the remaining iterations were kept to prevent autocorrelation (thinning strategy). The convergence of the MCMC chains was assessed by visual inspection of the chains and was tested using the Gelman-Rubin statistic (Plummer et al. 2006). A threshold value of 1.1 or less was assumed to suggest convergence (Gelman et al. 2013).

\section{Accuracy and precision of density estimates}

Generally, the usefulness and applicability of any method depends on the effort needed to achieve a target accuracy and precision. Here, the effort can be expressed as the number of cameras and/or the deployment time. Accordingly, each of the 90 simulated datasets (i.e., the count of animals detected per frame in 60 frames of 10 cameras) was submitted to 60 analyses (i.e., the $i^{\text {th }}$ analysis included the first frame to the $i^{\text {th }}$ frame). Therefore, a total number of 5,400 analyses was completed (6 archetypes, 15 independent simulations and 60 analyses per simulation).

Accuracy refers to how close an estimated parameter is to the true value. Here, it was defined as the scaled root mean squared error (SRMSE; Walther \& Moore, 2005):

$$
S R M S E=\frac{1}{D} \sqrt{\frac{1}{n} \sum_{\mathrm{j}=1}^{\mathrm{n}}\left(E_{\mathrm{j}}-D\right)^{2}},
$$

where $D$ was the true density, $E_{\mathrm{j}}$ was the median of the Bayesian posterior distribution, and $n$ was the number of replicates $(n=15)$.

Precision refers to the variability around the parameter estimate. Here, it was defined by the coefficient of variation ( $\mathrm{CV}$ ), which provides a scaled measure of precision, thus allowing a mean-independent comparison between archetypes (Walther \& Moore 2005):

$$
C V=\frac{100 \cdot S D}{\bar{E}},
$$
eqn 15

where $S D$ refers to the standard deviation, and $\bar{E}$ refers to the mean of the medians of the posterior distributions of the 15 replicated simulation experiments.

Finally, coverage was assessed by the percentage of analyses for which the $95 \%$ Bayesian credibility interval $(\mathrm{BCl})$ of the posterior distribution of the density estimate included the true value.

This article is protected by copyright. All rights reserved. 
A custom script in R was used for the full implementation (see Appendix S1 in Supporting Information). Furthermore, an additional simulation experiment was completed for assessing accuracy and precision when density is not spatially homogeneous (assumption iii) but patchy (see Appendix S2 for simulation details).

\section{Results}

\section{Selected archetypes and simulation experiments}

Nearly a thousand references $(n=980)$ provided data for at least two of the variables that beforehand were presumed to affect the quality of density estimates (density, body mass, speed and HR size), but data for all these variables were available for only 41 species (see Data Accessibility section and references list at Appendix S3). The correlation patterns between these variables were remarkable (see Fig. S1); further, the first two PCA axes explained $90 \%$ of the total variability (Fig. 1) and revealed two independent patterns: PC1 (explaining $64.0 \%$ of the variance) was well correlated with density, HR size and biomass, and PC2 (explaining $25.6 \%$ of the variance) was well correlated with speed. Based on these two independent axes, six well-contrasted archetypes were selected by visual inspection. The features of the archetypes were assigned from a freshwater fish (Campostoma anomalum (Rafinesque, 1820)), a marine fish (Chaetodon baronessa (Cuvier 1829)), a marine mammal (Monodon monoceros (Linnaeus, 1758)), a bird species (Brachyramphus marmoratus (Gmelin, 1789)), a terrestrial mammal (Alces alces (Linnaeus, 1758)) and a terrestrial reptile (Testudo graeca (Linnaeus, 1758)). Hereafter we refer them as freshwater fish-based (F/F-arch), marine fish-based (M/F-arch), marine mammal-based (M/M-arch), seabird-based (M/B-arch), terrestrial mammal-based (T/M-arch) and terrestrial reptile-based (T/R-arch) archetypes, respectively. The movement parameters for a $\operatorname{BRW}(k, \varepsilon$, and $\Delta t$ in eqn 3$)$ of these archetypes were estimated (eqns 5 and 6) from the values of speed and HR size reported in Table 1. Reliable values of camera radius (in relation to body mass) were obtained, ranging from $0.36 \mathrm{~m}$ to $630.8 \mathrm{~m}$ with $m=0.5$ (eqn 12). However, in the specific case of the $M / F-a r c h$, the archetype with the smallest HR size, we fixed the mean number of counts per camera up to 1.5 to increase the number of animals to be simulated (Table 1).

This article is protected by copyright. All rights reserved. 


\section{Assessing the success of density estimates}

After completing 5,400 analyses of the data from 90 simulation experiments, more than $90 \%$ of the $\mathrm{BCl}$ included the true value irrespective of the sampling effort and archetype, suggesting the proper retrieving of the density (Fig. 2, Table 2).

The general statement that accuracy and precision improve with an increase in sampling effort was supported in five out of the six archetypes (Fig. 2), but their densities were slightly underestimated. In those five cases, the averaged estimates of animal density did not show any substantial improvement after 1000-1500 frames. However, provided that the frame rate was archetype-specific, such a threshold implied different deployment times (Table 2). For example, for $\mathrm{M} / \mathrm{M}$-arch, accuracy improved $81 \%$ from low (50 frames, 14 hours of deployment time) to moderate sampling effort (1500 frames, 17 days) but only 15\% from moderate to high sampling effort (3000 frames, 35 days). The figures for precision were similar ( $86 \%$ and $16 \%$, respectively, Table 2 ).

When 10 cameras were deployed, the number of frames needed to reach a threshold of $10 \%$ accuracy ranged between 100 and 350 frames (Fig. 3a), which corresponded to deployment times ranging from 5 min to 75 days for each of the cameras, and depending on the archetype (Fig. 3b). After ranking the archetypes based on increasing deployment times, for F/F-arch, 9.3\% accuracy and 10\% precision were reached after 5 min 24 sec (250 frames); for M/B-arch, 9.6\% accuracy and 9.7\% precision were reached after $28.8 \mathrm{~min}$ (250 frames); for $\mathrm{M} / \mathrm{M}$-arch, $9.0 \%$ accuracy and $9.3 \%$ precision were reached after 28 hours $17 \mathrm{~min}$ (100 frames); for T/R-arch, $6.4 \%$ accuracy and $6.6 \%$ precision were reached after 54 days 10 hours 42 min (300 frames); for T/M-arch, 8.6\% accuracy and 8.4\% precision were reached after 75 days 7 hours $48 \mathrm{~min}$ (350 frames). When a high effort level was considered (i.e., 3000 frames or from 1 hour for F/F-arch up to 1.7 years to T/M-arch), the SRMSE was under $6.5 \%$ for all species, with the exception of the M/F-arch.

Accuracy and precision improved at different rates depending on the archetype because they crucially depended on how well the stationary distribution was reached at a given temporal scale. The more time a species took in exploring a relevant fraction of its HR, the more sampling time was needed to reach a target quality for the density estimates. A greater sampling effort was needed either when animals moved slowly (e.g., T/R-arch) or HR sizes were large in relation to the exploration rate (e.g., M/M-arch and T/M-arch) (Fig. 3b).

The case of the M/F-arch broke this general pattern. This species never reached a $10 \%$ error threshold for accuracy nor improved accuracy and precision after increasing the sampling effort (Fig. 3, Table 2). Likewise, the $\mathrm{BCl}$ of the posterior distribution was skewed in all cases

This article is protected by copyright. All rights reserved. 
(approximately $20 \%$ of the $\mathrm{BCls}$ were below the true value; Table 2 ). A plausible explanation for such a bias is discussed below.

Finally, it is noteworthy that the Bayesian approach proposed here is still precise and accurate even in the cases of spatial patchiness, whenever cameras are deployed beyond a distance ensuring the absence of spatial autocorrelation (Appendix S2).

\section{Discussion}

A generalization of the Hutchinson-Waser's postulate has been proposed here to estimate animal densities using terrestrial or underwater cameras (i.e., counts per frame) in species behaving according to the widely observed spatial behaviour that lead to the establishment of a HR area. The accuracy and precision reached for the density estimates after an affordable sampling effort provide further insight into the potential and comprehensiveness of the method. The results of the simulation experiments suggested that it would be sufficient to count animals in a few hundred frames adequately spaced in time to provide density estimates with SRMSEs smaller than $10 \%$. In contrast, the optimal time the cameras should be deployed (i.e., the optimal rate at which the frames should be counted) is species-dependent. For ten cameras, less than an hour of sampling (i.e., approximately 250 frames) was necessary for fish and bird-based archetypes, but more than 24 hours (i.e., between 100-350 frames) was needed for archetypes with larger HR areas, for which the time to cover the full HR area may range from a fortnight to more than one year (Table 1). The best estimates in terms of both accuracy and precision were obtained for animals with high densities, small HRs in relation to the exploration rate and large body mass. Overall, the results from the Bayesian implementation of the model demonstrate that density can be precisely and accurately estimated after an affordable sampling effort, but generally, the faster the animal covers its HR area, the less sampling time is needed. Nevertheless, some of the archetype-specific results should be interpreted with caution. For instance, in the case of the M/B-arch, density was accurately estimated after a very short survey time, which is related with its large speed and relatively small HR (Table 1). The speed value used in the simulation experiments has a relevant impact in the time needed for exploring a relevant fraction of the HR, thus a better reconstruction of the stationary probability can be obtained with smaller sampling effort for faster animals. However, speed definition is elusive and its magnitude is neither easily nor precisely estimated. Therefore, the patterns depicted in Fig 2 and 3 are suggesting a general guidance but species-specificities may modify the predicted effort needed for attaining a

This article is protected by copyright. All rights reserved. 
desired accuracy and precision. Hence, the relationship between the optimal sampling design (i.e., the optimal number of cameras, number of frames per camera and deployment time) and the space occupancy pattern of the animal may be complex. Therefore, it is strongly recommended to complete a pilot study (i.e., case-specific simulation experiments) including both, species-specificities and case-related constraints (e.g., budget available or technological limitations of the cameras) to assess whether the optimal settings are fulfilling or not the objectives in terms of expected accuracy and precision. For this purpose, R code in Appendix S1 can be easily adapted.

It should be noted, that a slightly systematic underestimation of the true density was detected in all simulations (Fig. 2). This bias may actually be an outcome of the simulation features instead of a methodological limitation. During the simulations, the virtual populations are bounded in the virtual scenario by the effective sampling area (eqn 13), and simulated animals could therefore move from inside the area to outside but not vice versa, leading to the aforementioned bias.

In spite of these uncertainties, the method proposed here has several advantages when comparing with increasingly popular video survey methods based on distilling metrics as $\mathrm{N}$-max (i.e., maximum number of animals in a given frame) as a proxy of abundance (Schobernd et al. 2014). From the conceptual side, the method proposed here provides an estimate for the absolute density, in contrast to the relative density provided by those alternative methods. In addition, animals must be only counted in a subsample of frames while for $\mathrm{N}$-max a full video must be analysed, which may represent a relevant difference in the time needed for processing the videos. Metrics as $N$-max are justified by the fact that they guaranties that the same animal is not recounted. Counting the same animal twice or even more times is a problem for non-instantaneous estimation methods developed for video surveys (Ward-Paige et al. 2010; Campbell et al. 2015). However, recounting does not imply any bias for the method proposed here. When animals move according to a HR space occupancy pattern, there exists a stationary probability density function of finding a given animal at a given position. Although we have focused on the particular case of the BRW (Börger et al. 2008), the same rationale may be extended to any other potential field and form of the stochastic term that renders a confined pattern of space use. Contrasting, markrecapture methods, which are based on a completely different rationale, incorporate repeated identification of the same individuals (e.g., Efford 2004; Royle \& Young 2008). Spatial explicit capture-recapture models aim to estimate the detection probability of any given animal, and that detectability can be disentangled from demographic processes (e.g. emigration/mortality and immigration/birth) given the history of captures (i.e., detections) of any given animal (Chandler

This article is protected by copyright. All rights reserved. 
\& Royle 2013). Certainly, our approach focuses in estimating animal density only. However, demographic rates could still be estimable by nesting the method proposed here into frameworks like those developed by fisheries assessment (Hilborn \& Walters 1992).

Another advantage of the method proposed here is that animal density can be estimated in a single visit to the sampled area. Simulation experiments were performed with a subsample of evenly distributed frames, being a convenience choice to facilitate the evaluation of temporal autocorrelation between frames. However, it may be any random subsample of the frames, after previously ensuring a safe between-frame period with temporal independence. Therefore, data from conventional camera trapping (i.e., camera is activated when an animal is present) can be easily adapted to this method. The benefit of motion-triggered camera surveys is that sampling time can be extended over longer periods of time and the resulting data can be broken down in smaller sampling units for analysis (Burton et al. 2015). Hence, the number of " 0 " data will be high but expected and with enough time the stationary probability density function of HR centres will be recovered. Certainly, other approaches can also obtain data from a single visit to the sampled location. For example, abundance can be inferred from occupancy models, which are primarily concerned with distribution (i.e., presence/absence), but in those cases individuals must be identified (recounting is a severe concern for the method (Royle 2004; Guillera-Arroita 2017)), and repeat surveys within the single visit are required (Marques et al. 2017).

Another advantage of the proposed method is that, neither stringent assumptions nor precise data on movement features are needed to estimate densities (but to define the optimal sampling settings some rough idea is required, Appendix S1). When using wildlife video recording methods, specific models that relate animal density with encounter rates have been widely considered: the random encounter model (REM), derived from ideal gas models (Hutchinson \& Waser 2007), and a closely related approach based on the Formozov-MalyshevPereleshin (FMP) formula (Stephens et al. 2006; Keeping \& Pelletier 2014), for which animal speed is assumed to be known (Rowcliffe et al. 2008; Cusack et al. 2015). A drawback of the REM and FMP is the non-instantaneous nature of encounters, which account for the speedrelated bias (Ward-Paige et al. 2010). Also, based on the encounter rates (i.e., detections are equivalent to encounters), distance sampling methods record distances from the observation point (typically line transects) to any detected animal (Buckland et al. 2001) and claim reasonable accuracy only if animals move at less than half the speed of the observer (WardPaige et al. 2010). Thus, in general, encounter-related methods are highly dependent on the assumptions on how animals move (Rowcliffe et al. 2008; Keeping \& Pelletier 2014; Glennie et al. 2015; Cusack et al. 2015). Different alternatives for circumventing this problem have been

This article is protected by copyright. All rights reserved. 
proposed (Hutchinson \& Waser 2007; Ward-Paige et al. 2010; Lucas et al. 2015). In some cases (e.g., line transects and camera traps), the encounter rate has been directly assumed to be a proxy for animal abundance (Marsden et al. 2016); in others, some assumptions are made regarding the movement pattern (Boschetti \& Vanderklift 2015).

In contrast, the raw input for the method proposed here consists of instantaneous observations (e.g., frames from a video survey) that are not directly affected by speed (Hutchinson \& Waser 2007). Nevertheless, accuracy and precision depend indirectly on movement features when sampling effort is constant. As stated before, HR behaviour is the result of a dynamic process in space and time (Börger et al. 2008); thus, species-specific sampling times are needed to successfully fit the stationary probability density function of finding an animal at a given position.

Some limitations of the method proposed here are related with departures from the implicit assumptions. Concerning the first assumption (i.e., the probability of detection is one), density estimation can be biased when imperfect detectability is not accounted for. When detection probability is known with high precision, robust density estimates will be obtained after changing $\lambda$ (averaged counts) by $p \lambda$ in eqn 11 (i.e., $N_{i j} \sim$ Poisson $\left.\left(p^{*} \lambda_{j}\right)\right)$, where $p$ is the detection probability. However when $p$ is known with some uncertainty, such uncertainty should be properly propagated, which deserves further derivations out of the scope of this contribution. Imperfect detectability may be related with technical limitations of the camera (e.g., resolution in pixels, detection zone, trigger speed, etc.), object properties (e.g., animal size, crypsis) or environmental heterogeneity. In practice, the combination of all those factors will define a case-specific optimal sampling area (Rovero et al. 2013) and preliminary trials are highly advisable to fit the technical limitations of the camera, the species specificities and the area actually surveyed (e.g., Martin et al. 2012). For example, it is expected that bigger animals could be efficiently detected and sampled using a bigger detection area of the camera. Similarly, common sense suggests maximizing the area surveyed at lower densities. On the other hand, partial detectability due to environmental factors (e.g., plant cover or 3D environmental heterogeneity, turbidity of waters (Hannah \& Blume 2016) or fog, etc.) could be solved by incorporating covariate effects on a detection probability function (Dénes et al. 2015). Those covariate effects has been successfully implemented in other approaches (MacKenzie et al. 2002; Fewster et al. 2009; Joseph et al. 2009). Concerning the second assumption (i.e., animals move independently from one another), when animals do not move independently (e.g., shoals or herds), density estimates should be referred to groups and not directly to individuals. Other complex and overspread behaviours as territoriality implies that animal movement depends on the density of conspecifics, thus further derivation of

This article is protected by copyright. All rights reserved. 
appropriate probability density functions (Potts \& Lewis 2014) should be needed in those cases.

Patchiness represents a genuine violation of the third assumption (i.e., the HR centres are randomly but homogeneously distributed). However, the mixed model proposed (eqn 11) would be able to address patchy distributions whenever a large number of cameras is deployed at appropriate between-cameras distance. A pilot study could solve the problem of finding the optimal distance between cameras to minimize spatial autocorrelation in patchy scenarios (Appendix S2). Similarly, animal density may change along an environmental gradient (e.g., depth-related distributional range), but incorporating environmental covariates into the model is straightforward, as well. Nevertheless, all these particular cases deserve further attention.

The case of the M/F-arch demonstrates one of the potential limitations of the model in relation to the third assumption. This archetype aimed to represent species with both small HR size and low density. The distribution of counts per frame showed striking differences between cameras: some cameras overlapped with the HR of one (or a few) animal(s), which will be detected most of the time, while others watched an area without HR centres. This fact is worsened when the detection area of the camera is relatively small, which was the case for the M/F-arch (7.6 times its HR size; Table 1). Therefore, although the HR centres were homogeneously distributed at a broad spatial scale, they were not at the scale of the detection area of the cameras. In contrast, F/F-arch aimed to represent species with small HR size (similar to M/F-arch) but that were very abundant (405 times the density of M/F-arch; Table 1). In this case, although the detection area was smaller, each camera was able to detect several animals, the stationary probability density function was properly reconstructed, and the density estimates were accurate and precise. Therefore, the case of the M/F-arch is not actually a modelling problem but may represent an inappropriate sampling design, which could be improved by either increasing the number of cameras and eventually decreasing the deployment time or increasing the detection area of the camera whenever technically possible. Again, this type of problem would be easily detected with a pilot experiment (Appendix S1).

Finally, it is well known that movement parameters vary between individuals of the same population (Alós, Palmer \& Arlinghaus 2012; Alós et al. 2016), and within individuals with time (i.e., age) due to changes in territory quality (Tao et al. 2016). The rationale of the method relies on the possibility of reconstructing the stationary probability density function by averaging over space the positions of identical animals in terms of movement features; thus,

This article is protected by copyright. All rights reserved. 
the outcomes of between-individual variability in movement characteristics should be also considered in the future.

In summary, absolute density may be accurately estimated for a great number of animals across taxa displaying HR behaviour from counting animals in frames adequately spaced in time, and after an affordable case-specific sampling effort, in a video recording survey. Notwithstanding the comprehensiveness of the method proposed here, a call of caution is necessary regarding the potential drawbacks listed above, and its merits and generality should be further explored.

\section{Acknowledgments}

ACC was supported by a FPU predoctoral fellowship (ref.FPU13/01440) from the Spanish Ministry of Education, Culture and Sports (MECD). JA was supported by a Juan de la Cierva postdoctoral grant (ref.FJCl-2014-21239) from the Spanish Ministry of Economy and Competitiveness (MINECO). This work was funded by R+D project PHENOFISH (ref.CTM201569126-C2-1-R; MINECO) and is a contribution of the Joint Research Unit IMEDEA-LIMIA. We thank the highly constructive and useful comments made by anonymous reviewers in an early version of the manuscript.

\section{Authors' contributions}

MP and SB conceived the ideas and all authors designed methodology; ACC performed simulations; ACC, MP and SB analysed the data; ACC, MP and JA led the writing of the manuscript. All authors contributed critically to the drafts and gave final approval for publication.

\section{Data Accessibility}

Data associated this manuscript are available from the Dryad Digital Repository: https://doi.org/ 10.5061/dryad.m605m (Campos-Candela et al. 2017).

\section{References}

Aguzzi, J., Doya, C., Tecchio, S., De Leo, F.C., Azzurro, E., Costa, C., Sbragaglia, V., Del Río, J., Navarro, J., Ruhl, H.A., Company, J.B., Favali, P., Purser, A., Thomsen, L. \& Catalán, I.A. (2015) Coastal observatories for monitoring of fish behaviour and their responses to

This article is protected by copyright. All rights reserved. 
environmental changes. Reviews in Fish Biology and Fisheries, 25, 463-483.

Alós, J., Palmer, M. \& Arlinghaus, R. (2012) Consistent Selection towards Low Activity Phenotypes When Catchability Depends on Encounters among Human Predators and Fish. PLOS ONE, 7, e48030.

Alós, J., Palmer, M., Balle, S. \& Arlinghaus, R. (2016) Bayesian State-Space Modelling of Conventional Acoustic Tracking Provides Accurate Descriptors of Home Range Behavior in a Small-Bodied Coastal Fish Species. PLoS ONE, 11, e0154089.

Assis, J., Claro, B., Ramos, A., Boavida, J. \& Serrão, E.A. (2013) Performing fish counts with a wide-angle camera, a promising approach reducing divers' limitations. Journal of Experimental Marine Biology and Ecology, 445, 93-98.

Bacheler, N.M., Schobernd, C.M., Schobernd, Z.H., Mitchell, W.A., Berrane, D.J., Kellison, G.T. \& Reichert, M.J.M. (2013) Comparison of trap and underwater video gears for indexing reef fish presence and abundance in the southeast United States. Fisheries Research, 143, 8188.

Boom, B.J., He, J., Palazzo, S., Huang, P.X., Beyan, C., Chou, H.-M., Lin, F.-P., Spampinato, C. \& Fisher, R.B. (2014) A research tool for long-term and continuous analysis of fish assemblage in coral-reefs using underwater camera footage. Ecological Informatics, 23, 83-97.

Börger, L., Dalziel, B.D. \& Fryxell, J.M. (2008) Are there general mechanisms of animal home range behaviour? A review and prospects for future research. Ecology Letters, 11, 637650.

Boschetti, F. \& Vanderklift, M.A. (2015) How the movement characteristics of large marine predators influence estimates of their abundance. Ecological Modelling, 313, 223-236.

Buckland, S.T., Anderson, D.R., Burnham, K.P., Laake, J.L., Borchers, D.L. \& Thomas, L. (2001) Introduction to Distance Sampling: Estimating Abundance of Biological Populations. Oxford University Press.

Burton, A.C., Neilson, E., Moreira, D., Ladle, A., Steenweg, R., Fisher, J.T., Bayne, E. \& Boutin, S. (2015) REVIEW: Wildlife camera trapping: a review and recommendations for linking surveys to ecological processes. Journal of Applied Ecology, 52, 675-685.

Campbell, M.D., Pollack, A.G., Gledhill, C.T., Switzer, T.S. \& DeVries, D.A. (2015) Comparison of relative abundance indices calculated from two methods of generating video count data. Fisheries Research, 170, 125-133.

Campos-Candela, Andrea; Palmer, Miquel; Balle, Salvador; Alós, Josep (2017) Data from: A camera-based method for estimating absolute density in animals displaying home range behaviour. Dryad Digital Repository. https://doi.org/ 10.5061/dryad.m605m

This article is protected by copyright. All rights reserved. 
Chandler, R.B. \& Royle, J.A. (2013) Spatially explicit models for inference about density in unmarked or partially marked populations. The Annals of Applied Statistics, 7, 936-954.

Cusack, J.J., Swanson, A., Coulson, T., Packer, C., Carbone, C., Dickman, A.J., Kosmala, M., Lintott, C. \& Rowcliffe, J.M. (2015) Applying a random encounter model to estimate lion density from camera traps in Serengeti National Park, Tanzania. The Journal of Wildlife Management, 79, 1014-1021.

Dell, A.I., Bender, J.A., Branson, K., Couzin, I.D., de Polavieja, G.G., Noldus, L.P.J.J., PérezEscudero, A., Perona, P., Straw, A.D., Wikelski, M. \& Brose, U. (2014) Automated imagebased tracking and its application in ecology. Trends in Ecology \& Evolution, 29, 417-428.

Dénes, F. V., Silveira, L.F. \& Beissinger, S.R. (2015) Estimating abundance of unmarked animal populations: accounting for imperfect detection and other sources of zero inflation. Methods in Ecology and Evolution, 6, 543-556.

Díaz-Gil, C., Louise Smee, S., Cotgrove, L., Follana-Berná, G., Hinz, H., Marti-Puig, P., Grau, A., Palmer, M. \& Catalán, I.A. (2017) Using stereoscopic video cameras to evaluate seagrass meadows nursery function in the Mediterranean. Marine Biology, 137.

Dubkov, A.A. \& Spagnolo, B. (2007) Langevin Approach to Levy Flights in Fixed Potentials: Exact Results for Stationary Probability Distributions. Acta Physica Polonica B, 38, 1745-1758.

Efford, M. (2004) Density estimation in live-trapping studies. Oikos, 106, 598-610.

Fewster, R.M., Buckland, S.T., Burnham, K.P., Borchers, D.L., Jupp, P.E., Laake, J.L. \& Thomas, L. (2009) Estimating the encounter rate variance in distance sampling. Biometrics, 65, 22536.

Gardiner, C.W. (1990) Handbook of Stochastic Methods for Physics, Chemistry and the Natural Sciences, Second Edi. Springer, Berlin.

Gelman, A., Carlin, J.B., Stern, H.., Dunson, D.B., Vehtari, A. \& Rubin, D.B. (2013) Bayesian Data Analysis, Third Edit (eds F Dominici, JJ Faraway, M Tanner, and J Zidek). Taylor \& Franci Group, LLC, United States.

Glennie, R., Buckland, S.T. \& Thomas, L. (2015) The Effect of Animal Movement on Line Transect Estimates of Abundance. PLOS ONE, 10, e0121333.

Goebel, M.E., Perryman, W.L., Hinke, J.T., Krause, D.J., Hann, N.A., Gardner, S. \& LeRoi, D.J. (2015) A small unmanned aerial system for estimating abundance and size of Antarctic predators. Polar Biology, 38, 619-630.

Guillera-Arroita, G. (2017) Modelling of species distributions, range dynamics and communities under imperfect detection: advances, challenges and opportunities. Ecography, 40, 281295.

This article is protected by copyright. All rights reserved. 
Hannah, R.W. \& Blume, M.T.O. (2016) Variation in the Effective Range of a Stereo-Video Lander in Relation to Near-Seafloor Water Clarity, Ambient Light and Fish Length. Marine and Coastal Fisheries, 8, 62-69.

Hilborn, R. \& Walters, C.J. (1992) Quantitative fisheries stock assessment: choice, dynamics, and uncertainty. Rev Fish Biol Fish, 2, 177-178.

Hodgson, J.C., Baylis, S.M., Mott, R., Herrod, A. \& Clarke, R.H. (2016) Precision wildlife monitoring using unmanned aerial vehicles. Scientific Reports, 6, 22574.

Hussey, N.E., Kessel, S.T., Aarestrup, K., Cooke, S.J., Cowley, P.D., Fisk, A.T., Harcourt, R.G., Holland, K.N., Iverson, S.J., Kocik, J.F., Mills Flemming, J.E. \& Whoriskey, F.G. (2015) Aquatic animal telemetry: A panoramic window into the underwater world. Science, 348, 1255642.

Hutchinson, J.M.C. \& Waser, P.M. (2007) Use, misuse and extensions of "ideal gas" models of animal encounter. Biological Reviews, 82, 335-359.

Joseph, L.N., Elkin, C., Martin, T.G. \& Possingham, H.P. (2009) Modeling abundance using Nmixture models: the importance of considering ecological mechanisms. Ecological Applications, 19, 631-642.

Keeping, D. \& Pelletier, R. (2014) Animal density and track counts: understanding the nature of observations based on animal movements. PLOS ONE, 9, e96598.

Kie, J.G., Matthiopoulos, J., Fieberg, J., Powell, R.A., Cagnacci, F., Mitchell, M.S., Gaillard, J.-M. \& Moorcroft, P.R. (2010) The home-range concept: are traditional estimators still relevant with modern telemetry technology? Philosophical Transactions of the Royal Society B: Biological Sciences, 365, 2221-2231.

Linchant, J., Lisein, J., Semeki, J., Lejeune, P. \& Vermeulen, C. (2015) Are unmanned aircraft systems (UASs) the future of wildlife monitoring? A review of accomplishments and challenges. Mammal Review, 45, 239-252.

Lucas, T.C.D., Moorcroft, E.A., Freeman, R., Rowcliffe, J.M. \& Jones, K.E. (2015) A generalised random encounter model for estimating animal density with remote sensor data. Methods in Ecology and Evolution, 6, 500-509.

MacKenzie, D.I., Nichols, J.D., Lachman, G.B., Droege, S., Royle, J.A. \& Langtimm, C.A. (2002) Estimating Site Occupancy Rates When Detection Probabilities Are Less Than One. Ecology, 83, 2248-2255.

Mallet, D. \& Pelletier, D. (2014) Underwater video techniques for observing coastal marine biodiversity: A review of sixty years of publications (1952-2012). Fisheries Research, 154, $44-62$.

This article is protected by copyright. All rights reserved. 
Marques, T.A., Thomas, L., Kéry, M., Buckland, S.T., Borchers, D.L., Rexstad, E., Fewster, R.M., MacKenzie, D.I., Royle, J.A., Guillera-Arroita, G., Handel, C.M., Pavlacky, D.C. \& Camp, R.J. (2017) Model-based approaches to deal with detectability: a comment on Hutto (2016a). Ecological Applications, 27, 1694-1698.

Marsden, S.J., Loqueh, E., Takuo, J.M., Hart, J.A., Abani, R., Ahon, D.B., Annorbah, N.N.D., Johnson, R. \& Valle, S. (2016) Using encounter rates as surrogates for density estimates makes monitoring of heavily-traded grey parrots achievable across Africa. Oryx, 50, 617625.

Martin, J., Edwards, H.H., Burgess, M.A., Percival, H.F., Fagan, D.E., Gardner, B.E., Ortega-Ortiz, J.G., Ifju, P.G., Evers, B.S. \& Rambo, T.J. (2012) Estimating distribution of hidden objects with drones: from tennis balls to manatees. PLOS ONE, 7, e38882.

Matabos, M., Piechaud, N., De Montigny, F., Sarradin, P.-M., Sarrazin, J. \& Rochet, M.-J. (2015) The VENUS cabled observatory as a method to observe fish behaviour and species assemblages in a hypoxic fjord, Saanich Inlet (British Columbia, Canada). Canadian Journal of Fisheries and Aquatic Sciences, 72, 24-36.

Van Moorter, B., Visscher, D., Benhamou, S., Börger, L., Boyce, M.S. \& Gaillard, J.M. (2009) Memory keeps you at home: A mechanistic model for home range emergence. Oikos, 118, 641-652.

Moya, Ó., Mansilla, P., Madrazo, S., Igual, J., Rotger, A., Romano, A. \& Tavecchia, G. (2015) APHIS: A new software for photo-matching in ecological studies ÓscarMoya. Ecological Informatics, 27, 64-70.

Oksanen, J., Blanchet, F.G., Friendly, M., Kindt, R., Legendre, P., Mcglinn, D., Minchin, P.R., Hara, R.B.O., Simpson, G.L., Solymos, P., Stevens, M.H.H. \& Szoecs, E. (2016) Package "vegan" (Version 2.4-0). URL https://cran.r-project.org, https://github.com/vegandevs/vegan.

Owen-Smith, N. \& Martin, J. (2015) Identifying Space Use at Foraging Arena Scale within the Home Ranges of Large Herbivores. PLOS ONE, 10, e0128821.

Palmer, M., Balle, S., March, D., Alós, J. \& Linde, M. (2011) Size estimation of circular home range from fish mark-release-(single)-recapture data: case study of a small labrid targeted by recreational fishing. Marine Ecology Progress Series, 430, 87-97.

Pelletier, D., Leleu, K., Mou-Tham, G., Guillemot, N. \& Chabanet, P. (2011) Comparison of visual census and high definition video transects for monitoring coral reef fish assemblages. Fisheries Research, 107, 84-93.

Plummer, M., Best, N., Cowles, K. \& Vines, K. (2006) CODA: convergence diagnosis and output analysis for MCMC. $R$ News, 6, 7-11.

This article is protected by copyright. All rights reserved. 
Potts, J.R. \& Lewis, M.A. (2014) How do animal territories form and change? Lessons from 20 years of mechanistic modelling. Proceedings of the Royal Society B, 281, 20140231.

Rovero, F., Zimmermann, F., Berzi, D. \& Meek, P. (2013) “Which camera trap type and how many do I need?" A review of camera features and study designs for a range of wildlife research applications. Hystrix, 24, 148-156.

Rowcliffe, J.M., Field, J., Turvey, S.T. \& Carbone, C. (2008) Estimating animal density using camera traps without the need for individual recognition. Journal of Applied Ecology, 45, $1228-1236$.

Royle, J.A. (2004) N-Mixture Models for Estimating Population Size from Spatially Peplicated Counts. Biometrics, 60, 108-115.

Royle, J.A. \& Young, K. V. (2008) A hierarchical model for spatial capture-recapture data. Ecology, 89, 2281-2289.

Schobernd, Z.H., Bacheler, N.M., Conn, P.B. \& Trenkel, V. (2014) Examining the utility of alternative video monitoring metrics for indexing reef fish abundance. Canadian Journal of Fisheries and Aquatic Sciences, 71, 464-471.

Stephens, P.A., Zaumyslova, O.Y., Miquelle, D.G., Myslenkov, A.I. \& Hayward, G.D. (2006) Estimating population density from indirect sign: track counts and the FormozovMalyshev-Pereleshin formula. Animal Conservation, 9, 339-348.

Su, Y.-S. \& Yajima, M. (2015) Package "R2jags" (Version:0.5-7). URL: http://cran.rproject.org/package=R2jags .

Tao, Y., Börger, L. \& Hastings, A. (2016) Dynamic Range Size Analysis of Territorial Animals: An Optimality Approach. The American Naturalist, 000-000.

Vas, E., Lescroël, A., Duriez, O., Boguszewski, G. \& Grémillet, D. (2015) Approaching birds with drones: first experiments and ethical guidelines. Biology letters, 11, 20140754.

Walther, B.A. \& Moore, J.L. (2005) The concepts of bias, precision and accuracy, and their use in testing the performance of species richness estimators, with a literature review of estimator performance. Ecography, 28, 815-829.

Ward-Paige, C., Mills Flemming, J. \& Lotze, H.K. (2010) Overestimating Fish Counts by NonInstantaneous Visual Censuses: Consequences for Population and Community Descriptions. PLOS ONE, 5, e11722.

Williams, K., Rooper, C.N. \& Towler, R. (2010) Use of stereo camera systems for assessment of rockfish abundancein untrawlable areas and for recording pollock behavior during midwater trawls. Fishery Bulletin, 108, 352-362.

This article is protected by copyright. All rights reserved. 


\section{Supporting Information}

Additional Supporting Information may be found in the online version of this article:

Appendix S1. Guidelines to apply the model to a case study.

Appendix S2. Evaluating accuracy and precision in patchy landscapes.

Appendix S3. Supplementary bibliography. .

Fig. S1. Bivariate correlations between densities, body mass, speed and HR size.

\section{Figures}

Figure1

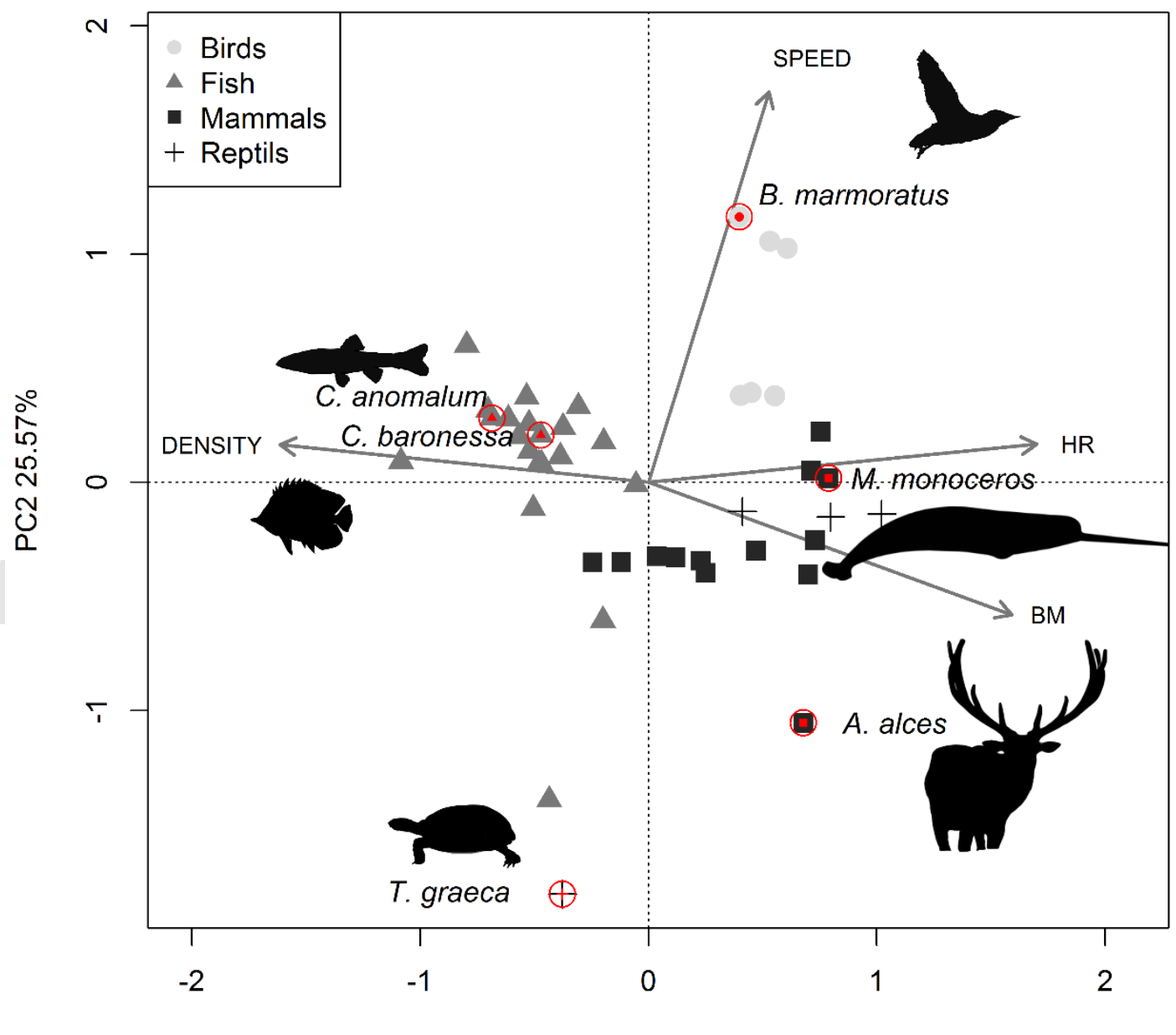

PC1 64.04\%

This article is protected by copyright. All rights reserved. 
Figure 2
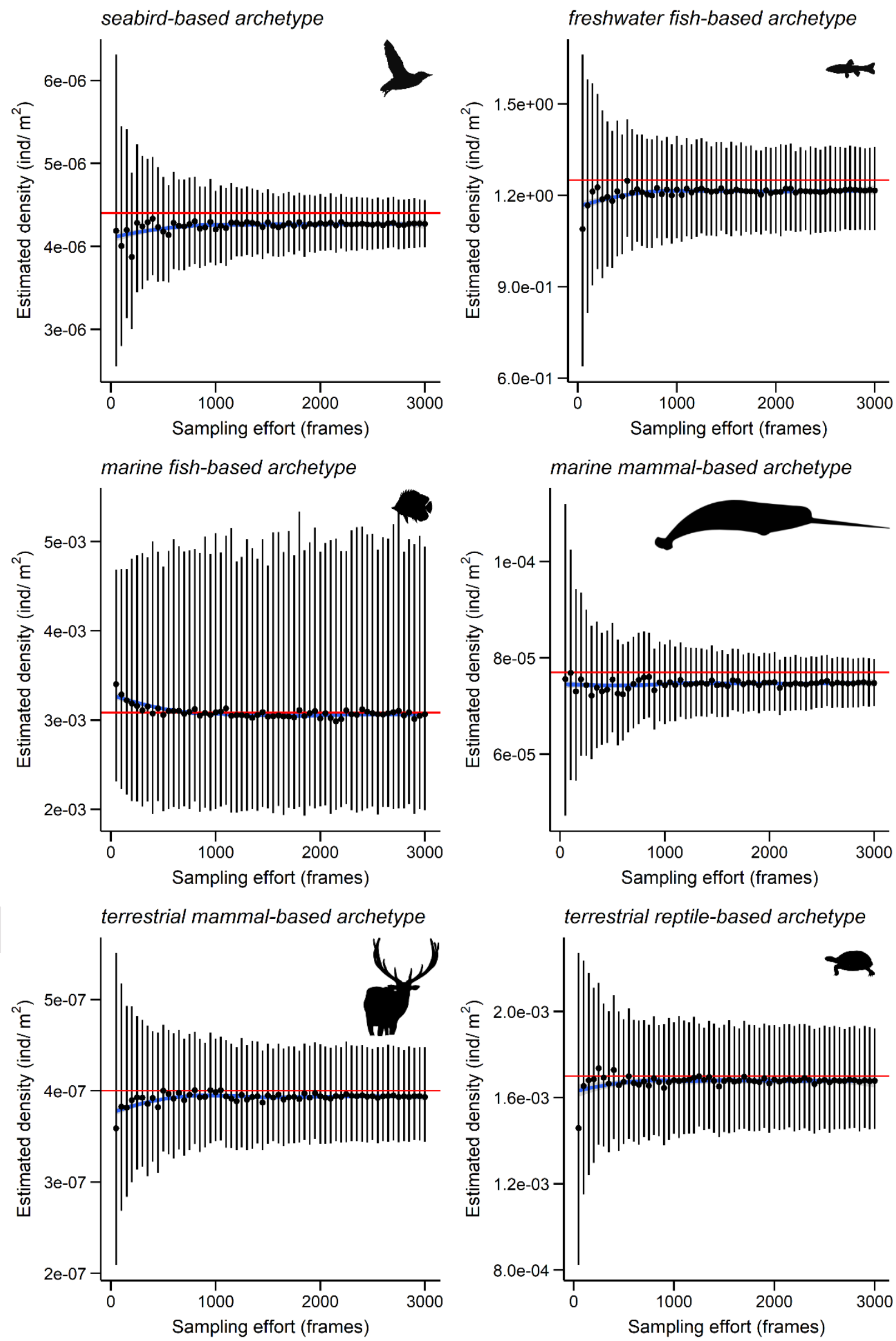

This article is protected by copyright. All rights reserved. 
Figure 3
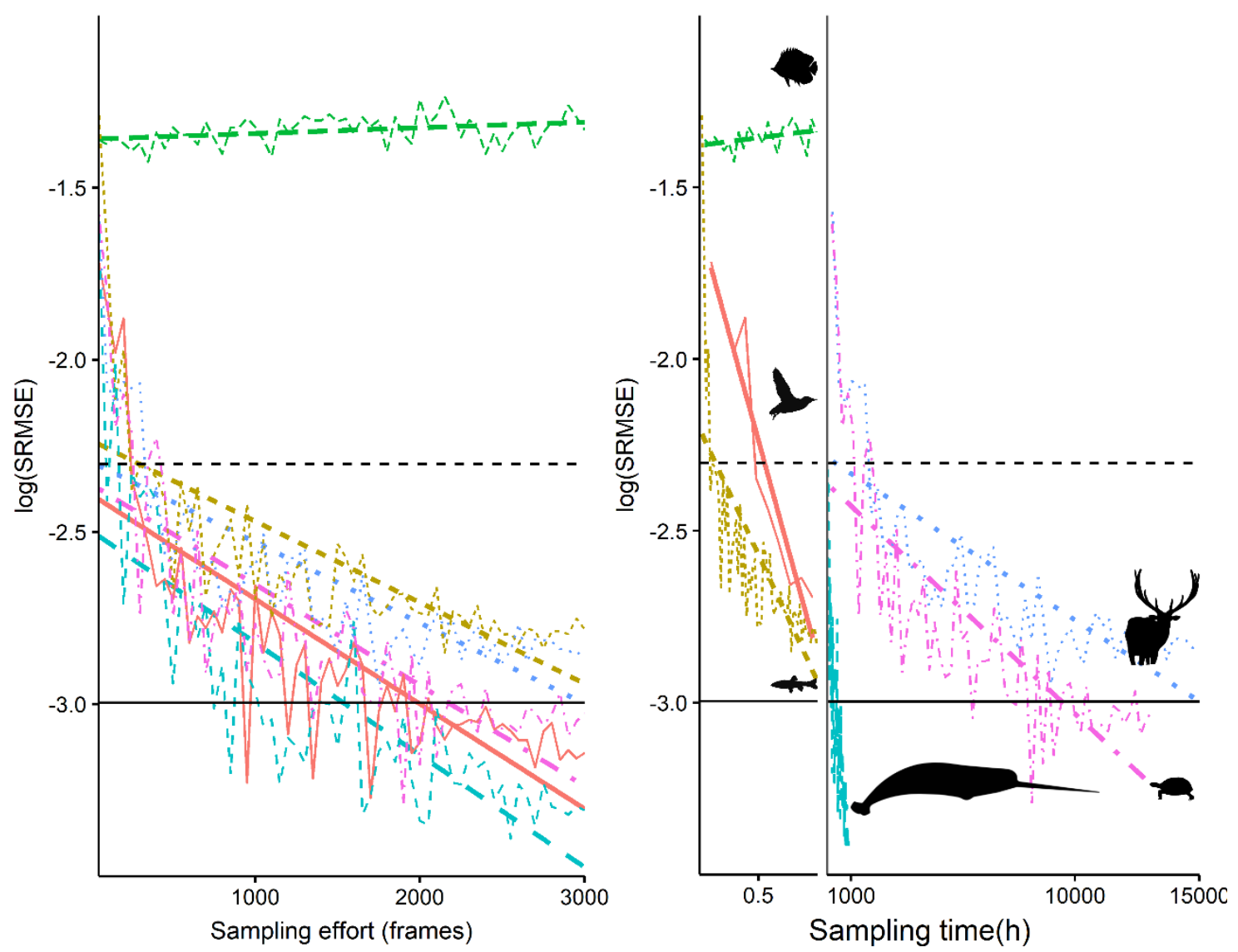

- seabird-based arch - " marine fish-based arch " " terrestrial mammal-based arch
$-=$ freshwater fish-based arch - marine mammal-based arch " - terrestrial reptile-based arch

This article is protected by copyright. All rights reserved. 
Figure 1. Bi-plot of the principal component analysis (PCA) performed with four variables: HR size $\left(\mathrm{km}^{2}\right)$, speed $(\mathrm{m} / \mathrm{s})$, biomass $(\mathrm{g})$ and density (animals $\left./ \mathrm{km}^{2}\right)$. Variables are plotted as vectors, and the angle between them can be interpreted as the bivariate correlation in the multivariate space. The first two PCA components explained $89.6 \%$ of the total variance. The dataset was composed of 43 species: birds $(n=6)$, freshwater $(n=8)$ and marine $(n=12)$ fish, marine $(n=3)$ and terrestrial $(n=10)$ mammals and marine $(n=3)$ and terrestrial $(n=1)$ reptiles. Figures correspond to selected archetypes for simulation analyses.

Figure 2. Estimated density with increasing sampling effort (number of frames analysed by 10 cameras). Mean values (black points) and 95\% BCls of the medians from 15 posterior distributions of the density estimates. The red line corresponds to the true value, and the blue line indicates bias. Vertical axes are not scaled.

Figure 3. Log-transformed scaled root mean squared error (SRMSE) of density estimates and linear fit lines with increasing sampling effort: frames analysed by 10 cameras (panel a) and deployment time for each camera (panel b). Black dotted and continuous lines correspond to threshold values of $10 \%$ and $5 \%$ of the SRMSE, respectively.

This article is protected by copyright. All rights reserved. 
This article has been accepted for publication and undergone full peer review but has not been through the copyediting, typesetting, pagination and proofreading process, which may lead to differences between this version and the Version of Record. Please cite this article as doi:

10.1111/1365-2656.12787

This article is protected by copyright. All rights reserved. 


\section{Tables}

Table 1. Input data, simulation features and estimated movement parameters for the selected archetypes. S/G-arch refers to the code for each archetype including the system $(\mathrm{S})$ in which animals live: marine $(\mathrm{M})$, freshwater $(\mathrm{F})$ or terrestrial $(\mathrm{T})$; and the group (G) animals belong: birds (B), fish (F), mammals (M) and reptiles (R). BM: biomass (g), HR: area of the HR $\left(\mathrm{km}^{2}\right)$, D: density (animals $/ \mathrm{km}^{2}$ ) and S: speed (m/s)were obtained from the literature (Data Accessibility section and Appendix S3). Simulation features: DR: detection radius of the camera (m); SEA: size of the scenario (km²); N: number of simulated animals; and $\mathrm{P}$ : minimum $\Delta t$ steps between consecutive frames to avoid temporal autocorrelation. The movement parameters $(k, \varepsilon$ and $\Delta t)$ were estimated from HR and

\begin{tabular}{|c|c|c|c|c|c|c|c|c|c|c|c|c|}
\hline S/G-arch & Species & $\begin{array}{l}\text { BM } \\
(\mathrm{g})\end{array}$ & $\begin{array}{c}\mathrm{HR} \\
\left(\mathrm{Km}^{2}\right)\end{array}$ & $\begin{array}{c}\mathrm{D} \\
\left(\mathrm{ind} / \mathrm{Km}^{2}\right)\end{array}$ & $\begin{array}{c}\mathrm{S} \\
(\mathrm{m} / \mathrm{s})\end{array}$ & $\begin{array}{l}\text { DR } \\
\text { (m) }\end{array}$ & $\begin{array}{c}\text { SEA } \\
\left(\mathrm{Km}^{2}\right)\end{array}$ & $\mathrm{N}$ & $\mathrm{P}$ & $\begin{array}{c}k \\
(1 / \mathrm{sec})\end{array}$ & $\begin{array}{c}\varepsilon \\
\left(\mathrm{m}^{2} / \mathrm{s}\right)\end{array}$ & $\begin{array}{l}\Delta t \\
(\mathrm{~s})\end{array}$ \\
\hline $\mathrm{M} / \mathrm{B}$ & Brachyramphus marmoratus & 220 & 127 & 4.4 & 22.6 & 190 & 171.5 & 755 & 1 & 0.001445 & $19,499.4$ & 69.2 \\
\hline $\mathrm{F} / \mathrm{F}$ & Campostoma anomalum & 34 & $3.510^{-05}$ & $1,250,000$ & 0.629 & 0.36 & $5.510^{-05}$ & 68 & 10 & 0.076609 & 0.28490 & 1.3 \\
\hline $\mathrm{M} / \mathrm{F}$ & Chaetodon baronessa & 27.63 & $6.310^{-05}$ & $3,083.3$ & 0.5555 & 12.4 & 0.001 & 4 & 15 & 0.050333 & 0.33821 & 1.9 \\
\hline $\mathrm{M} / \mathrm{M}$ & Monodon monoceros & 938,126 & 10,400 & 77 & 1.39 & 45.5 & $3,320.9$ & 255,709 & 1 & $9.8210^{-06}$ & $10,852.8$ & 10,182 \\
\hline $\mathrm{T} / \mathrm{M}$ & Alces alces & 461,901 & 82 & 0.4 & 0.03379 & 630.8 & 131.8 & 53 & 5 & $2.6910^{-06}$ & 23.4283 & 37,189 \\
\hline T/R & Testudo graeca & 450 & 0.0171 & 1,700 & 0.00058 & 9.7 & 0.03 & 47 & 5 & $3.1910^{-06}$ & 0.00579 & 31,360 \\
\hline
\end{tabular}

This article is protected by copyright. All rights reserved. 

Table 2. Accuracy (SRMSE), precision (CV\%) and coverage (proportion of 95\% BCls including the true density value) for the selected archetypes. Accuracy and precision are referred to as the medians of the Bayesian posterior distributions. Three levels of sampling effort (number of frames obtained in total by ten cameras) are shown. Sampling time refers to the archetypespecific period of time needed by a camera to obtain the number of frames indicated.

Archetypes are ordered by accuracy.

\begin{tabular}{|c|c|c|c|c|c|}
\hline & $\begin{array}{l}\text { Number } \\
\text { of frames }\end{array}$ & $\begin{array}{l}\text { Sampling time } \\
\text { (hours) }\end{array}$ & $\begin{array}{l}\text { Accuracy: } \\
\text { SRMSE }\end{array}$ & $\begin{array}{l}\text { Precision: } \\
\text { CV\% }\end{array}$ & Coverage \\
\hline \multicolumn{6}{|l|}{$M / M-\operatorname{arch}$} \\
\hline & 50 & 14.14 & 0.195 & 20.5 & $1 \pm 0$ \\
\hline & 1500 & 424.25 & 0.044 & 2.87 & $1 \pm 0$ \\
\hline & 3000 & 848.51 & 0.037 & 2.40 & $0.87 \pm 0.35$ \\
\hline \multicolumn{6}{|l|}{$M / B-\operatorname{arch}$} \\
\hline & 50 & 0.10 & 0.180 & 18.8 & $1 \pm 0$ \\
\hline & 1500 & 2.88 & 0.052 & 4.93 & $0.93 \pm 0.26$ \\
\hline & 3000 & 5.77 & 0.043 & 3.40 & $0.87 \pm 0.35$ \\
\hline \multicolumn{6}{|l|}{$T / R-\operatorname{arch}$} \\
\hline & 50 & 217.78 & 0.206 & 18.1 & $0.93 \pm 0.26$ \\
\hline & 1500 & $6,533.35$ & 0.056 & 5.75 & $1 \pm 0$ \\
\hline & 3000 & $13,066.70$ & 0.048 & 4.90 & $1 \pm 0$ \\
\hline \multicolumn{6}{|l|}{$T / M$-arch } \\
\hline & 50 & 258.26 & 0.208 & 20.8 & $1 \pm 0$ \\
\hline & 1500 & $7,747.81$ & 0.058 & 5.92 & $0.93 \pm 0.26$ \\
\hline & 3000 & $15,495.63$ & 0.058 & 5.88 & $0.93 \pm 0.26$ \\
\hline \multicolumn{6}{|l|}{$F / F-\operatorname{arch}$} \\
\hline & 50 & 0.02 & 0.275 & 28.9 & $0.80 \pm 0.41$ \\
\hline & 1500 & 0.54 & 0.079 & 7.78 & $0.80 \pm 0.41$ \\
\hline & 3000 & 1.09 & 0.062 & 5.90 & $0.93 \pm 0.26$ \\
\hline \multicolumn{6}{|l|}{$M / F-\operatorname{arch}$} \\
\hline & 50 & 0.04 & 0.256 & 22.0 & $0.87 \pm 0.35$ \\
\hline & 1500 & 1.24 & 0.270 & 28.4 & $0.87 \pm 0.35$ \\
\hline & 3000 & 2.48 & 0.264 & 27.5 & $0.80 \pm 0.41$ \\
\hline
\end{tabular}

This article is protected by copyright. All rights reserved. 